\title{
Five models for lepton mixing
}

\author{
P.M. Ferreira, ${ }^{a, b}$ L. Lavoura ${ }^{c}$ and P.O. Ludl ${ }^{d}$
}

${ }^{a}$ Instituto Superior de Engenharia de Lisboa, 1959-007 Lisbon, Portugal

${ }^{b}$ Centre for Theoretical and Computational Physics, University of Lisbon 1649-003 Lisbon, Portugal

${ }^{c}$ Technical University of Lisbon, Instituto Superior Técnico, CFTP, 1049-001 Lisbon, Portugal

${ }^{d}$ University of Vienna, Faculty of Physics, Boltzmanngasse 5, A-1090 Vienna, Austria

E-mail: ferreira@cii.fc.ul.pt, balio@cftp.ist.utl.pt, patrick.ludl@univie.ac.at

ABStRaCt: We produce five flavour models for the lepton sector. All five models fit perfectly well — at the $1 \sigma$ level — the existing data on the neutrino mass-squared differences and on the lepton mixing angles. The models are based on the type I seesaw mechanism, on a $\mathbb{Z}_{2}$ symmetry for each lepton flavour, and either on a (spontaneously broken) symmetry under the interchange of two lepton flavours or on a (spontaneously broken) $C P$ symmetry incorporating that interchange - or on both symmetries simultaneously. Each model makes definite predictions both for the scale of the neutrino masses and for the phase $\delta$ in lepton mixing; the fifth model also predicts a correlation between the lepton mixing angles $\theta_{12}$ and $\theta_{23}$.

Keywords: Neutrino Physics, Discrete and Finite Symmetries 


\section{Contents}

1 Introduction $\quad 1$

2 The matrix $A \quad 3$

$\begin{array}{lll}3 & \text { Model } 1 & 4\end{array}$

$\begin{array}{lll}4 & \text { Model } 2 & 7\end{array}$

5 Models 3, 4, and $5 \quad 9$

$\begin{array}{lll}6 & \text { Observable predictions } & 10\end{array}$

$\begin{array}{lll}7 & \text { Summary } & 13\end{array}$

\section{Introduction}

The problem of explaining the observed features of lepton mixing became significantly more awkward with the recent definitive (more than $5 \sigma$ ) indications for a non-zero mixing angle $\theta_{13}[1-3]$. These indications have rendered obsolete the idea of a $\mu-\tau$ interchange symmetry in the neutrino mass matrix [4]. Moreover, a not so well-established $(2 \sigma)$ indication that the mixing angle $\theta_{23}$ is not maximal ${ }^{1}$ suggests that the idea of a $C P$ symmetry incorporating the $\mu-\tau$ interchange [8] should be discarded too.

At this juncture, one may either search for other ideas or try and somehow modify the approaches mentioned in the previous paragraph in order to make them compatible with the data. In this paper we take the latter path. Specifically, while in the models of refs. $[4,9]$ the neutrino Dirac mass matrix $M_{D}$ was of the form $\operatorname{diag}(a, b, b)$ and in the model of ref. [8] it was $\operatorname{diag}\left(a, b, b^{*}\right)$, in this paper we present models where $M_{D}=\operatorname{diag}(a, b, c)$. We shall see that this still allows us to retain some predictive power. In order to enhance that power, we also apply in this paper an idea originally proposed in ref. [9], which allows us to obtain one vanishing off-diagonal matrix element in the inverse of the light-neutrino Majorana mass matrix $M$ (in the basis where the charged-lepton mass matrix is diagonal).

In general, the matrix $M$, which is symmetric, contains nine physical parameters: the six moduli of its matrix elements and three rephasing-invariant phases. Those nine parameters correspond to nine observables: three neutrino masses, three mixing angles, one Dirac phase $(\delta)$ in the mixing, and two Majorana phases among the three neutrino masses. The first four models that we present in this paper have only five physical parameters and are thus comparable, in predictive power, to models or Ansätze with two 'texture

\footnotetext{
${ }^{1}$ In this paper we use exclusively the global fit of the neutrino-oscillation data by Fogli et al. [5]. Other fits are contained in refs. [6, 7].
} 
zeros' $[10,11]$. Those models are able to predict $\delta$ and the absolute scale of the neutrino masses. $^{2}$ Moreover, our models provide excellent fits to the existing data on the three mixing angles and on the two neutrino mass-squared differences. Our fifth model has only four physical parameters and is able to predict one correlation between two mixing angles.

All the models that we propose in this paper utilize the type I seesaw mechanism with three right-handed neutrinos. Thus, the leptonic multiplets in our models are the following:

- Three right-handed charged-lepton gauge-SU(2) singlets $\alpha_{R}$, where $\alpha$ may take the values $e, \mu$, and $\tau$;

- three right-handed neutrino singlets $\nu_{\alpha R}$;

- three left-handed doublets $D_{\alpha L}=\left(\nu_{\alpha L}, \alpha_{L}\right)^{T}$.

Our models also have a very simple scalar sector:

- Two Higgs doublets $\phi_{1,2}=\left(\phi_{1,2}^{+}, \phi_{1,2}^{0}\right)^{T}$. Their conjugate doublets are $\tilde{\phi}_{1,2}=$ $\left(\phi_{1,2}^{0}{ }^{*},-\phi_{1,2}^{-}\right)^{T}$

- two real gauge singlets, $\chi_{1}$ and $\chi_{2}$.

It is useful to compare the models in this paper with other recent models and Ansätze which attempt to explain the nonzero $\theta_{13}$ and non-maximal $\theta_{23}$. Our models 3,4 , and 5 in this paper are based on an $e^{-} \tau$ interchange symmetry (embedded, in models 4 and 5 , in a generalized $C P$ symmetry) and therefore would never, even if that symmetry went unbroken in $M_{D}$, predict $\theta_{13}=0$. In our models 1 and 2 there is a $\mu-\tau$ interchange symmetry (embedded in model 1 in a $D_{4}$ non-Abelian symmetry, and in model 2 in a generalized $C P$ symmetry), ${ }^{3}$ which is, however, broken in the neutrino Dirac mass matrix $M_{D}$. By contrast, many other models start from neither $\mu-\tau$, nor $e-\tau$, nor $e-\mu$ interchange symmetries. One approach focuses on 'texture zeros' in $M$; this was recently advocated in ref. [13] and numerically developed in ref. [14]. Alternatively one may use non-Abelian symmetries like $A_{4}$-which do not contain the above-mentioned interchange symmetries and had proven useful [15] for constructing models with vanishing $\theta_{13}$-while relinquishing additional assumptions in such a way that finally a nonzero $\theta_{13}$ emerges; an example of this approach is ref. [16]. Or else one may use those non-Abelian symmetries in altogether novel ways, see for instance ref. [17]. Another approach tries to find completely novel predictions for $\theta_{13}$ and $\theta_{23}$ by having recourse to much larger non-Abelian flavour symmetry groups together with carefully picked vacuum states; three very recent examples are given in refs. [18-20], papers with related approaches are in refs. [21-23].

The outline of this paper is as follows. In section 2 we introduce a matrix $A$, derived from the matrix $M$ but which does not suffer from the rephasing ambiguity of the latter; it turns out that most predictions of our models can be stated in terms of $A$-matrix elements. In section 3 we present our first model, which uses a $\mu-\tau$ interchange symmetry.

\footnotetext{
${ }^{2}$ We omit predictions for the two Majorana phases, since they are, in general, experimentally irrelevant.

${ }^{3}$ For a short review on the $\mu-\tau$ interchange symmetry, see ref. [12].
} 
In section 4 we present our second model, which uses a $C P$ symmetry incorporating the $\mu-\tau$ interchange. In section 5 we note that in both previous models one may use an $e-\tau$ interchange instead of the $\mu-\tau$ interchange and still obtain a perfect fit to the data; we moreover note that, by combining the $e^{-\tau}$ interchange symmetry with the corresponding generalized $C P$ symmetry, one obtains a fifth model that is still compatible with the data. In section 6 we elaborate, by means of a fit to the phenomenological data and of scatter plots, the predictions of our models. We briefly summarize our achievements in section 7 .

\section{The matrix $A$}

Let

$$
M=\left(\begin{array}{lll}
a & f & e \\
f & b & d \\
e & d & c
\end{array}\right)
$$

be a symmetric $3 \times 3$ matrix. We assume $M$ to be invertible. Then,

$$
M^{-1}=\frac{1}{\operatorname{det} M}\left(\begin{array}{ccc}
b c-d^{2} & d e-c f & d f-b e \\
d e-c f & a c-e^{2} & e f-a d \\
d f-b e & e f-a d & a b-f^{2}
\end{array}\right) .
$$

We define the matrix $A$, which is a symmetric $3 \times 3$ matrix too, through

$$
A_{\alpha \beta}=M_{\alpha \beta}\left(M^{-1}\right)_{\alpha \beta},
$$

where we do not imply summation over $\alpha$ and $\beta{ }^{4}$ Then,

$$
A=\frac{1}{\operatorname{det} M}\left(\begin{array}{ccc}
a b c-a d^{2} & d e f-c f^{2} & d e f-b e^{2} \\
d e f-c f^{2} & a b c-b e^{2} & d e f-a d^{2} \\
d e f-b e^{2} & d e f-a d^{2} & a b c-c f^{2}
\end{array}\right)
$$

An advantage of the matrix $A$ over the matrix $M$ is that, when $M$ gets rephased through

$$
M_{\alpha \beta} \rightarrow e^{i\left(\psi_{\alpha}+\psi_{\beta}\right)} M_{\alpha \beta},
$$

the matrix $A$ remains invariant.

Since

$$
\operatorname{det} M=a b c+2 d e f-a d^{2}-b e^{2}-c f^{2},
$$

the matrix $A$ in eq. (2.4) satisfies

$$
\sum_{\alpha=1}^{3} A_{\alpha \beta}=1
$$

for any value of $\beta$. It follows from eq. (2.7) that only three elements of $A$ are linearly independent. Therefore $A$ has only six parameters: three moduli and three phases. ${ }^{5}$

\footnotetext{
${ }^{4}$ In this section, Greek-letter indices have range $\{1,2,3\}$. In the other sections of this paper, $M$ is interpreted as the neutrino mass matrix and those indices indicate the lepton flavours $e, \mu$ and $\tau$.

${ }^{5}$ The original matrix $M$, after allowing for the rephasing (2.5), has nine parameters: six moduli and three phases. So, $A$ contains less information than $M$.
} 
Equation (2.7) has several consequences. Let $\alpha \neq \beta \neq \gamma \neq \alpha$. Then,

$$
A_{\gamma \alpha}=A_{\gamma \beta} \Leftrightarrow A_{\alpha \alpha}=A_{\beta \beta},
$$

which holds because $A_{\alpha \alpha}=1-A_{\beta \alpha}-A_{\gamma \alpha}, A_{\beta \beta}=1-A_{\alpha \beta}-A_{\gamma \beta}$, and $A$ is symmetric. Another consequence is

$$
A_{\gamma \alpha}=A_{\gamma \beta}^{*} \Rightarrow A_{\gamma \gamma}=A_{\gamma \gamma}^{*},
$$

which holds because $A_{\gamma \gamma}=1-A_{\alpha \gamma}-A_{\beta \gamma}$. Still another consequence of eq. (2.7) is

$$
A_{\gamma \alpha}=A_{\gamma \beta}^{*} \wedge A_{\alpha \alpha}=A_{\beta \beta}^{*} \Rightarrow A_{\alpha \beta}=A_{\alpha \beta}^{*} .
$$

Finally, one further consequence of eq. (2.7) is

$$
A_{\gamma \alpha}=A_{\gamma \beta}^{*} \wedge A_{\alpha \beta}=A_{\alpha \beta}^{*} \Rightarrow A_{\alpha \alpha}=A_{\beta \beta}^{*} \text {. }
$$

\section{Model 1}

Our first model has lepton flavour symmetry $D_{4}$. It is directly inspired by the model of ref. [9]; as a matter of fact, it is a simplification thereof, since in ref. [9] the symmetry group is $D_{4} \times \mathbb{Z}_{2}$ (and since ref. [9] has three Higgs doublets while we need only two). Here we dispense with the $\mathbb{Z}_{2}$ since we want to treat in exactly the same fashion the charged-lepton and the neutrino Yukawa couplings to the Higgs doublets.

A handy introduction to the groups $D_{n}$ and to their representations can be found in ref. [24]. We recall that $D_{4}$ is generated, in its defining doublet irreducible representation, by the matrices

$$
B=\left(\begin{array}{cc}
1 & 0 \\
0 & -1
\end{array}\right) \quad \text { and } \quad D=\left(\begin{array}{ll}
0 & 1 \\
1 & 0
\end{array}\right) .
$$

The group $D_{4}$ has four inequivalent singlet representations,

$$
\mathbf{1}_{p q}: \quad B \rightarrow p 1, D \rightarrow q 1, \quad \text { where } \quad p, q \in\{+,-\} .
$$

The groups $D_{n}$ have been frequently used as flavour symmetry groups; for a review see ref. [25]. In particular, $D_{4}$ was utilized in a large variety of models, some of which are collected in refs. [26-29].

Our model contains the $D_{4}$ doublets

$$
\left(\begin{array}{c}
D_{\mu L} \\
D_{\tau L}
\end{array}\right), \quad\left(\begin{array}{c}
\mu_{R} \\
\tau_{R}
\end{array}\right), \quad\left(\begin{array}{c}
\nu_{\mu R} \\
\nu_{\tau R}
\end{array}\right), \quad \text { and } \quad\left(\begin{array}{c}
\chi_{1} \\
\chi_{2}
\end{array}\right),
$$

while the Higgs doublet $\phi_{2}$ is a $\mathbf{1}_{+-}$of $D_{4}$. The remaining fields, viz. $D_{e L}, e_{R}, \nu_{e R}$, and $\phi_{1}$ are $D_{4}$-invariant. In particular this means that our model 1 has a $\mathbb{Z}_{2}$ interchange symmetry generated by the matrix $D$ in eq. (3.1),

$$
\mathbb{Z}_{2}^{(\text {int })}: D_{\mu L} \leftrightarrow D_{\tau L}, \mu_{R} \leftrightarrow \tau_{R}, \nu_{\mu R} \leftrightarrow \nu_{\tau R}, \chi_{1} \leftrightarrow \chi_{2}, \phi_{2} \rightarrow-\phi_{2} .
$$


This symmetry is broken spontaneously at the low (Fermi or lower $\left.{ }^{6}\right)$ scale when $\phi_{2}^{0}$ acquires a vacuum expectation value (VEV). There is, besides, the $\mathbb{Z}_{2}$ symmetry generated by the matrix $B$ in eq. (3.1),

$$
\mathbb{Z}_{2}^{(\tau)}: D_{\tau L}, \tau_{R}, \nu_{\tau R}, \chi_{2} \text { change sign. }
$$

This symmetry is broken spontaneously at the high (seesaw) scale when $\chi_{2}$ acquires a VEV.

It so happens that our model also has — just as the model of ref. [9] — two accidental $\mathbb{Z}_{2}$ symmetries,

$$
\begin{aligned}
& \mathbb{Z}_{2}^{(e)}: D_{e L}, e_{R}, \nu_{e R}, \chi_{1}, \chi_{2} \text { change sign, } \\
& \mathbb{Z}_{2}^{(\mu)}: D_{\mu L}, \mu_{R}, \nu_{\mu R}, \chi_{1} \text { change sign. }
\end{aligned}
$$

These accidental symmetries are also broken at the seesaw scale when $\chi_{1,2}$ acquire VEVs. We parameterize those VEVs as

$$
\left\langle 0\left|\chi_{1}\right| 0\right\rangle \equiv U \cos \vartheta, \quad\left\langle 0\left|\chi_{2}\right| 0\right\rangle \equiv U \sin \vartheta
$$

The Lagrangian of Majorana mass terms is then of the form

$$
\mathcal{L}_{\text {Maj }}=-\frac{1}{2}\left[m \bar{\nu}_{e R} C \bar{\nu}_{e R}^{T}+m^{\prime}\left(\bar{\nu}_{\mu R} C \bar{\nu}_{\mu R}^{T}+\bar{\nu}_{\tau R} C \bar{\nu}_{\tau R}^{T}\right)\right]+\text { H.c. }
$$

The Yukawa Lagrangian is

$$
\begin{aligned}
\mathcal{L}_{\text {Yuk }}= & -y_{1} \bar{D}_{e L} e_{R} \phi_{1} \\
& -y_{2}\left(\bar{D}_{\mu L} \mu_{R}+\bar{D}_{\tau L} \tau_{R}\right) \phi_{1}-y_{3}\left(\bar{D}_{\mu L} \mu_{R}-\bar{D}_{\tau L} \tau_{R}\right) \phi_{2} \\
& -y_{4} \bar{D}_{e L} \nu_{e R} \tilde{\phi}_{1} \\
& -y_{5}\left(\bar{D}_{\mu L} \nu_{\mu R}+\bar{D}_{\tau L} \nu_{\tau R}\right) \tilde{\phi}_{1}-y_{6}\left(\bar{D}_{\mu L} \nu_{\mu R}-\bar{D}_{\tau L} \nu_{\tau R}\right) \tilde{\phi}_{2} \\
& -y_{7}\left(\chi_{1} \bar{\nu}_{\mu R}+\chi_{2} \bar{\nu}_{\tau R}\right) C \bar{\nu}_{e R}^{T}+\text { H.c. },
\end{aligned}
$$

where $C$ is the charge-conjugation matrix in Dirac space. So the charged-lepton mass matrix is diagonal,

$$
M_{\ell}=\operatorname{diag}\left(y_{1} v_{1}, y_{2} v_{1}+y_{3} v_{2}, y_{2} v_{1}-y_{3} v_{2}\right),
$$

where $v_{k}=\left\langle 0\left|\phi_{k}^{0}\right| 0\right\rangle$ for $k=1,2$. The neutrino Dirac mass matrix is diagonal too:

$$
M_{D}=\operatorname{diag}(a, b, c),
$$

\footnotetext{
${ }^{6}$ Our Higgs doublets $\phi_{1,2}$ are not necessarily the ones which couple to the quarks. Their VEVs may be much lower (and their masses much higher) than the Fermi scale, suppressed for instance through a type II seesaw mechanism [30]. Alternatively, our model 1 may be viewed as an ordinary two-Higgs-doublet model furnished with a $\mathbb{Z}_{2}$ symmetry under which $\phi_{2} \rightarrow-\phi_{2}$; that symmetry may apply to the quark sector in a variety of ways [31], for instance by inverting the signs of the right-handed down-type quarks. It has been shown [32-35] that these models are quite capable of describing the phenomenology and experimental constraints that arise from the recent observations of a Higgs-like particle [36, 37].
} 
where $a=y_{4}^{*} v_{1}, b=y_{5}^{*} v_{1}+y_{6}^{*} v_{2}$, and $c=y_{5}^{*} v_{1}-y_{6}^{*} v_{2}$. The Majorana mass matrix of the right-handed neutrinos is given by

$$
M_{R}=\left(\begin{array}{ccc}
m & y_{7} U \cos \vartheta & y_{7} U \sin \vartheta \\
y_{7} U \cos \vartheta & m^{\prime} & 0 \\
y_{7} U \sin \vartheta & 0 & m^{\prime}
\end{array}\right) .
$$

The expression for the effective light-neutrino Majorana mass matrix is ${ }^{7}$

$$
M=-M_{D}^{T} M_{R}^{-1} M_{D}
$$

Since $M_{D}$ is diagonal and $\left(M_{R}\right)_{\mu \tau}=0$, it immediately follows that

$$
\left(M^{-1}\right)_{\mu \tau}=0
$$

Taking into account the imposed symmetries, one finds the scalar potential

$$
\begin{aligned}
V= & \sum_{k=1}^{2}\left[\mu_{k} \phi_{k}^{\dagger} \phi_{k}+\lambda_{k}\left(\phi_{k}^{\dagger} \phi_{k}\right)^{2}\right] \\
& +\lambda_{3} \phi_{1}^{\dagger} \phi_{1} \phi_{2}^{\dagger} \phi_{2}+\lambda_{4} \phi_{1}^{\dagger} \phi_{2} \phi_{2}^{\dagger} \phi_{1}+\left[\lambda_{5}\left(\phi_{1}^{\dagger} \phi_{2}\right)^{2}+\text { H.c. }\right] \\
& +\left(\mu_{\chi}+\sum_{k=1}^{2} \bar{\lambda}_{k} \phi_{k}^{\dagger} \phi_{k}\right)\left(\chi_{1}^{2}+\chi_{2}^{2}\right)+\lambda_{\chi}\left(\chi_{1}^{2}+\chi_{2}^{2}\right)^{2} \\
& +\lambda_{\chi}^{\prime} \chi_{1}^{2} \chi_{2}^{2}+\left(\lambda \phi_{1}^{\dagger} \phi_{2}+\text { H.c. }\right)\left(\chi_{1}^{2}-\chi_{2}^{2}\right) .
\end{aligned}
$$

We assume that $U$ is at the high scale, while $v=\left(\left|v_{1}\right|^{2}+\left|v_{2}\right|^{2}\right)^{1 / 2}$ lies at the low scale. ${ }^{8}$ The vacuum potential for $U$ and $\vartheta$ is

$$
V_{0}=\left(\mu_{\chi}+\sum_{k=1}^{2} \bar{\lambda}_{k}\left|v_{k}\right|^{2}\right) U^{2}+\lambda_{\chi} U^{4}+\frac{\lambda_{\chi}^{\prime}}{4} U^{4} \sin ^{2} 2 \vartheta+2 \Re\left(\lambda v_{1}^{*} v_{2}\right) U^{2} \cos 2 \vartheta .
$$

Unless $\lambda$ is very large, of order $U^{2} / v^{2}$, the last term in the right-hand side of eq. (3.16) is much smaller than the term just before it and may be neglected. ${ }^{9}$ Then $\vartheta$ will be either $n \pi / 2$ or $(2 n+1) \pi / 4$ (with integer $n$ ) depending on whether $\lambda_{\chi}^{\prime}$ is, respectively, positive or negative. We assume that $\lambda_{\chi}^{\prime}$ is negative and that $\vartheta=\pi / 4$, i.e. that $\left\langle 0\left|\chi_{1}\right| 0\right\rangle=\left\langle 0\left|\chi_{2}\right| 0\right\rangle$. This equality holds to $\mathcal{O}\left(v^{2} / U^{2}\right)$ when $\lambda$ and $\lambda_{\chi}^{\prime}$ are of the same order.

\footnotetext{
${ }^{7}$ In section $2, M$ was a generic $3 \times 3$ symmetric, non-singular matrix. From now on, $M$ will specifically denote the light-neutrino Majorana mass matrix in the basis where the charged-lepton mass matrix is diagonal.

${ }^{8}$ This of course requires some finetuning in $V$. Namely, $\bar{\lambda}_{1,2}$ must be very small, of order $v^{2} / U^{2}$, in order that $\mu_{1}$ and $\mu_{2}$ do not receive corrections of seesaw magnitude when $\chi_{1,2}$ acquire VEVs. This is an ordinary 'hierarchy problem', to which we cannot offer a solution.

${ }^{9}$ This is the same approximation as in eq. (3.13), which neglects terms of order $v^{2} / U^{2}[38]$ too.
} 
Thus,

$$
M^{-1}=-\operatorname{diag}\left(\frac{1}{a}, \frac{1}{b}, \frac{1}{c}\right)\left(\begin{array}{ccc}
m & m^{\prime \prime} & m^{\prime \prime} \\
m^{\prime \prime} & m^{\prime} & 0 \\
m^{\prime \prime} & 0 & m^{\prime}
\end{array}\right) \operatorname{diag}\left(\frac{1}{a}, \frac{1}{b}, \frac{1}{c}\right),
$$

where $m^{\prime \prime}=y_{7} U / \sqrt{2}$. Hence,

$$
\begin{aligned}
& M=-\frac{1}{m^{\prime}\left(m m^{\prime}-2 m^{\prime \prime 2}\right)} \operatorname{diag}(a, b, c) \\
& \times\left(\begin{array}{ccc}
m^{\prime 2} & -m^{\prime} m^{\prime \prime} & -m^{\prime} m^{\prime \prime} \\
-m^{\prime} m^{\prime \prime} & m m^{\prime}-m^{\prime \prime 2} & m^{\prime \prime 2} \\
-m^{\prime} m^{\prime \prime} & m^{\prime \prime 2} & m m^{\prime}-m^{\prime \prime 2}
\end{array}\right) \operatorname{diag}(a, b, c) .
\end{aligned}
$$

It follows from eqs. (3.17) and (3.18) that ${ }^{10}$

$$
A_{e \mu}=A_{e \tau}
$$

We conclude that model 1 leads to two complex conditions on $M$, eqs. (3.14) and (3.19). Therefore, the matrix $M$ in this model contains five physical parameters, as opposed to nine physical parameters in the general case.

\section{Model 2}

In model 2 , the symmetry $\mathbb{Z}_{2}^{(\mathrm{int})}$ of model 1 is substituted by the $C P$ symmetry

$$
\begin{gathered}
\left\{\begin{array} { l } 
{ D _ { e L } ( x ) \rightarrow \gamma _ { 0 } C \overline { D } _ { e L } ^ { T } ( \overline { x } ) } \\
{ D _ { \mu L } ( x ) \rightarrow \gamma _ { 0 } C \overline { D } _ { \tau L } ^ { T } ( \overline { x } ) } \\
{ D _ { \tau L } ( x ) \rightarrow \gamma _ { 0 } C \overline { D } _ { \mu L } ^ { T } ( \overline { x } ) }
\end{array} \quad \left\{\begin{array}{l}
e_{R}(x) \rightarrow \gamma_{0} C \bar{e}_{R}^{T}(\bar{x}) \\
\mu_{R}(x) \rightarrow \gamma_{0} C \bar{\tau}_{R}^{T}(\bar{x}), \\
\tau_{R}(x) \rightarrow \gamma_{0} C \bar{\mu}_{R}^{T}(\bar{x})
\end{array}\right.\right. \\
\left\{\begin{array} { l } 
{ \nu _ { e R } ( x ) \rightarrow \gamma _ { 0 } C \overline { \nu } _ { e R } ^ { T } ( \overline { x } ) } \\
{ \nu _ { \mu R } ( x ) \rightarrow \gamma _ { 0 } C \overline { \nu } _ { \tau R } ^ { T } ( \overline { x } ) } \\
{ \nu _ { \tau R } ( x ) \rightarrow \gamma _ { 0 } C \overline { \nu } _ { \mu R } ^ { T } ( \overline { x } ) }
\end{array} \quad \left\{\begin{array}{l}
\chi_{1}(x) \rightarrow \chi_{2}(\bar{x}) \\
\chi_{2}(x) \rightarrow \chi_{1}(\bar{x}) \\
\phi_{1}(x) \rightarrow \phi_{1}^{*}(\bar{x}) \\
\phi_{2}(x) \rightarrow-\phi_{2}^{*}(\bar{x})
\end{array}\right.\right.
\end{gathered}
$$

where $x=(t, \vec{r})$ and $\bar{x}=(t,-\vec{r})$. Notice that, since $\chi_{1}$ and $\chi_{2}$ are gauge-singlet fields, they have no electroweak interactions, hence $C P$ may be arbitrarily defined for them; we have opted for $\chi_{1} \stackrel{C P}{\leftrightarrow} \chi_{2}$. Also notice that the Higgs doublets $\phi_{1}$ and $\phi_{2}$ transform with opposite signs under $C P$.

Besides, model 2 also has the symmetries $\mathbb{Z}_{2}^{(e)}, \mathbb{Z}_{2}^{(\mu)}$, and $\mathbb{Z}_{2}^{(\tau)}$ of model 1. Therefore, the symmetry features of model 2 cannot be readily described through a group, since they include a $C P$ symmetry that does not commute with the two usual symmetries $\mathbb{Z}_{2}^{(\mu)}$ and $\mathbb{Z}_{2}^{(\tau)}$. The theory of this kind of flavour symmetries has for example recently been investigated in ref. [39]; ${ }^{11}$ other recent examples of their utilization are refs. [43, 44].

\footnotetext{
${ }^{10} A_{\mu \mu}=A_{\tau \tau}$ also follows from eqs. (3.17) and (3.18) but is equivalent to eq. (3.19), cf. eq. (2.8).

${ }^{11}$ See also ref. [40]. Generalized $C P$ symmetries were pioneered in refs. [41, 42].
} 
We now have

$$
\mathcal{L}_{\text {Maj }}=-\frac{1}{2}\left[m \bar{\nu}_{e R} C \bar{\nu}_{e R}^{T}+m^{\prime} \bar{\nu}_{\mu R} C \bar{\nu}_{\mu R}^{T}+m^{\prime *} \bar{\nu}_{\tau R} C \bar{\nu}_{\tau R}^{T}\right]+\text { H.c. }
$$

where $m$ is real, and

$$
\begin{aligned}
\mathcal{L}_{\text {Yuk }}= & -y_{1} \bar{D}_{e L} e_{R} \phi_{1} \\
& -\left(y_{2} \bar{D}_{\mu L} \mu_{R}+y_{2}^{*} \bar{D}_{\tau L} \tau_{R}\right) \phi_{1}-\left(y_{3} \bar{D}_{\mu L} \mu_{R}-y_{3}^{*} \bar{D}_{\tau L} \tau_{R}\right) \phi_{2} \\
& -y_{4} \bar{D}_{e L} \nu_{e R} \tilde{\phi}_{1} \\
& -\left(y_{5} \bar{D}_{\mu L} \nu_{\mu R}+y_{5}^{*} \bar{D}_{\tau L} \nu_{\tau R}\right) \tilde{\phi}_{1}-\left(y_{6} \bar{D}_{\mu L} \nu_{\mu R}-y_{6}^{*} \bar{D}_{\tau L} \nu_{\tau R}\right) \tilde{\phi}_{2} \\
& -\left(y_{7} \chi_{1} \bar{\nu}_{\mu R}+y_{7}^{*} \chi_{2} \bar{\nu}_{\tau R}\right) C \bar{\nu}_{e R}^{T}+\text { H.c. },
\end{aligned}
$$

where $y_{1}$ and $y_{4}$ are real. So the charged-lepton mass matrix is once again diagonal,

$$
M_{\ell}=\operatorname{diag}\left(y_{1} v_{1}, y_{2} v_{1}+y_{3} v_{2}, y_{2}^{*} v_{1}-y_{3}^{*} v_{2}\right),
$$

just as the neutrino Dirac mass matrix, which is as in eq. (3.11) with $a=y_{4}^{*} v_{1}, b=$ $y_{5}^{*} v_{1}+y_{6}^{*} v_{2}$, and $c=y_{5} v_{1}-y_{6} v_{2}$. The Majorana mass matrix of the right-handed neutrinos is given by

$$
M_{R}=\left(\begin{array}{ccc}
m & y_{7} U \cos \vartheta & y_{7}^{*} U \sin \vartheta \\
y_{7} U \cos \vartheta & m^{\prime} & 0 \\
y_{7}^{*} U \sin \vartheta & 0 & m^{\prime *}
\end{array}\right)
$$

and eq. (3.14) still holds.

The scalar potential is

$$
\begin{aligned}
V= & \sum_{k=1}^{2}\left[\mu_{k} \phi_{k}^{\dagger} \phi_{k}+\lambda_{k}\left(\phi_{k}^{\dagger} \phi_{k}\right)^{2}\right] \\
& +\lambda_{3} \phi_{1}^{\dagger} \phi_{1} \phi_{2}^{\dagger} \phi_{2}+\lambda_{4} \phi_{1}^{\dagger} \phi_{2} \phi_{2}^{\dagger} \phi_{1}+\lambda_{5}\left[\left(\phi_{1}^{\dagger} \phi_{2}\right)^{2}+\text { H.c. }\right] \\
& +\left(\mu_{\chi}+\sum_{k=1}^{2} \bar{\lambda}_{k} \phi_{k}^{\dagger} \phi_{k}\right)\left(\chi_{1}^{2}+\chi_{2}^{2}\right)+\lambda_{\chi}\left(\chi_{1}^{2}+\chi_{2}^{2}\right)^{2} \\
& +\lambda_{\chi}^{\prime} \chi_{1}^{2} \chi_{2}^{2}+\left[\lambda\left(\phi_{1}^{\dagger} \phi_{2} \chi_{1}^{2}-\phi_{2}^{\dagger} \phi_{1} \chi_{2}^{2}\right)+\text { H.c. }\right],
\end{aligned}
$$

where $\lambda_{5}$ is real but $\lambda$ is in general complex. Once again, if $\lambda_{\chi}^{\prime}$ is negative then $\vartheta=\pi / 4$. Thus,

$$
M^{-1}=-\operatorname{diag}\left(\frac{1}{a}, \frac{1}{b}, \frac{1}{c}\right)\left(\begin{array}{ccc}
m & m^{\prime \prime} & m^{\prime \prime *} \\
m^{\prime \prime} & m^{\prime} & 0 \\
m^{\prime \prime *} & 0 & m^{\prime *}
\end{array}\right) \operatorname{diag}\left(\frac{1}{a}, \frac{1}{b}, \frac{1}{c}\right),
$$

where $m^{\prime \prime}=y_{7} U / \sqrt{2}$ Hence,

$$
\begin{aligned}
M^{-1}=-\frac{1}{m\left|m^{\prime}\right|^{2}}-2 \Re\left(m^{\prime *} m^{\prime \prime 2}\right) & \operatorname{diag}(a, b, c) \\
& \times\left(\begin{array}{ccc}
\left|m^{\prime}\right|^{2} & -m^{\prime *} m^{\prime \prime} & -m^{\prime} m^{\prime \prime *} \\
-m^{\prime *} m^{\prime \prime} & m m^{\prime *}-m^{\prime \prime * 2} & \left|m^{\prime \prime}\right|^{2} \\
-m^{\prime} m^{\prime \prime *} & \left|m^{\prime \prime}\right|^{2} & m m^{\prime}-m^{\prime \prime 2}
\end{array}\right) \operatorname{diag}(a, b, c) .
\end{aligned}
$$


Therefore this model predicts ${ }^{12}$

$$
A_{e \mu}=A_{e \tau}^{*} .
$$

So this model has once again five parameters, since it predicts the two complex eqs. (3.14) and (4.9).

\section{Models 3, 4, and 5}

In the models of the previous two sections we have made the following assignment for the lepton flavours: we have assumed that the lepton flavours being interchanged either by the symmetry $\mathbb{Z}_{2}^{\text {(int) }}$ of eq. (3.4) or by the $C P$ symmetry of eq. (4.1) are the $\mu$ and $\tau$ flavours. Two other choices are possible - the lepton flavours being interchanged might be either $e$ and $\mu$ or $e$ and $\tau$. We have tested those two other choices against the experimental data and have found that the second choice fits those data just as well as models 1 and 2 above. Thus, we define model 3 as predicting

$$
\begin{aligned}
\left(M^{-1}\right)_{e \tau} & =0 \\
A_{e \mu} & =A_{\mu \tau},
\end{aligned}
$$

and model 4 as predicting

$$
\begin{aligned}
\left(M^{-1}\right)_{e \tau} & =0, \\
A_{e \mu} & =A_{\mu \tau}^{*} .
\end{aligned}
$$

It is also possible to simultaneously impose invariance under $\mathbb{Z}_{2}^{(\text {int) }}$ of eq. (3.4) and the $C P$ symmetry of eq. (4.1). In that case,

- Equation (3.8) holds with $m$ and $m^{\prime}$ real, cf. eq. (4.2).

- Equation (3.9) holds with $y_{1-7}$ real, cf. eq. (4.3).

- Equation (3.15) holds with $\lambda_{5}$ and $\lambda$ real, cf. eq. (4.6).

So, finally one ends up with eq. (3.17) with $m, m^{\prime}$, and $m^{\prime \prime}$ real. The $C P$ symmetry may be broken spontaneously through VEVs $v_{1,2}$ with a relative complex phase and this makes $a, b$, and $c$ complex; but that has no relevance for our predictions, since $a, b, c$ drop out in the matrix $A$.

It turns out that the model delineated above is unable to correctly fit the present data. But the model in which the $e$ and $\mu$ flavours are interchanged relative to the above works fine; hence we define model 5 as predicting

$$
\begin{aligned}
\left(M^{-1}\right)_{e \tau} & =0, \\
A_{e \mu} & =A_{\mu \tau}, \\
A_{\mu \tau} & =A_{\mu \tau}^{*} .
\end{aligned}
$$

\footnotetext{
${ }^{12}$ It also predicts $A_{\mu \mu}=A_{\tau \tau}^{*}, A_{e e}=A_{e e}^{*}$, and $A_{\mu \tau}=A_{\mu \tau}^{*}$, but these three relations can be derived from eqs. (3.14) and (4.9), as was pointed out at the end of section 2.
} 


\section{Observable predictions}

We have fitted models $1-5$ of the previous sections to the phenomenological data of ref. [5]. It turns out that our models fit the data so perfectly that we were able to use just the $1 \sigma$ intervals given in that paper. Thus, we have required that

$$
\begin{aligned}
& m_{2}^{2}-m_{1}^{2} \in[7.32,7.80] \times 10^{-5} \mathrm{eV}^{2}, \\
& \sin ^{2} \theta_{12} \in[0.291,0.325], \\
& m_{3}^{2}-\frac{m_{1}^{2}+m_{2}^{2}}{2} \in[2.33,2.49] \times 10^{-3} \mathrm{eV}^{2}, \\
& \sin ^{2} \theta_{13} \in[0.0216,0.0266], \\
& \sin ^{2} \theta_{23} \in[0.365,0.410],
\end{aligned}
$$

in the case of a normal neutrino mass spectrum. If the neutrino mass spectrum is inverted, then the requirements (6.1a) and (6.1b) remain, but the requirements (6.1c)-(6.1e) are substituted by

$$
\begin{aligned}
& \frac{m_{1}^{2}+m_{2}^{2}}{2}-m_{3}^{2} \in[2.31,2.49] \times 10^{-3} \mathrm{eV}^{2}, \\
& \sin ^{2} \theta_{13} \in[0.0219,0.0267], \\
& \sin ^{2} \theta_{23} \in[0.370,0.431],
\end{aligned}
$$

respectively. In eqs. (6.1) and (6.2), $m_{1,2,3}$ are the neutrino masses and $\theta_{12,13,23}$ are the lepton mixing angles as defined in the standard parameterization in eq. (13.79) of ref. [45]. Notice that we have not used in our fits the constraints in ref. [5] for the Dirac phase $\delta$ of lepton mixing, which we chose instead to be an observable to be predicted by our models. For comparison, at the $1 \sigma$ level the authors of ref. [5] found

$$
\begin{array}{lll}
\delta \in[0.77 \pi, 1.36 \pi] & \Rightarrow & \cos \delta \in[-1,-0.43] \\
\delta \in[0.83 \pi, 1.47 \pi] & \Rightarrow & \cos \delta \in[-1,-0.09]
\end{array}
$$

for a normal and for an inverted spectrum, respectively.

We have found that all our models are able to fit the data either in eqs. (6.1) - for a normal mass spectrum - or in eqs. (6.2) - for an inverted mass spectrum - or in both of them - perfectly. In models 1 to 4, the phase space for the five observables appears, inside the intervals quoted in those equations, uniformly filled in all our scatter plots, so that no prediction for any of those observables looks warranted. On the other hand, predictions for the overall neutrino mass scale and for the phase $\delta$ are possible. Another quantity that one may predict is the effective mass $m_{\beta \beta} \equiv\left|M_{e e}\right|$, which is relevant for neutrinoless double beta decay. In model 5 , moreover, the angles $\theta_{12}$ and $\theta_{23}$ are correlated and significantly restricted relative to the intervals in eqs. (6.1b) and (6.2c), respectively. 

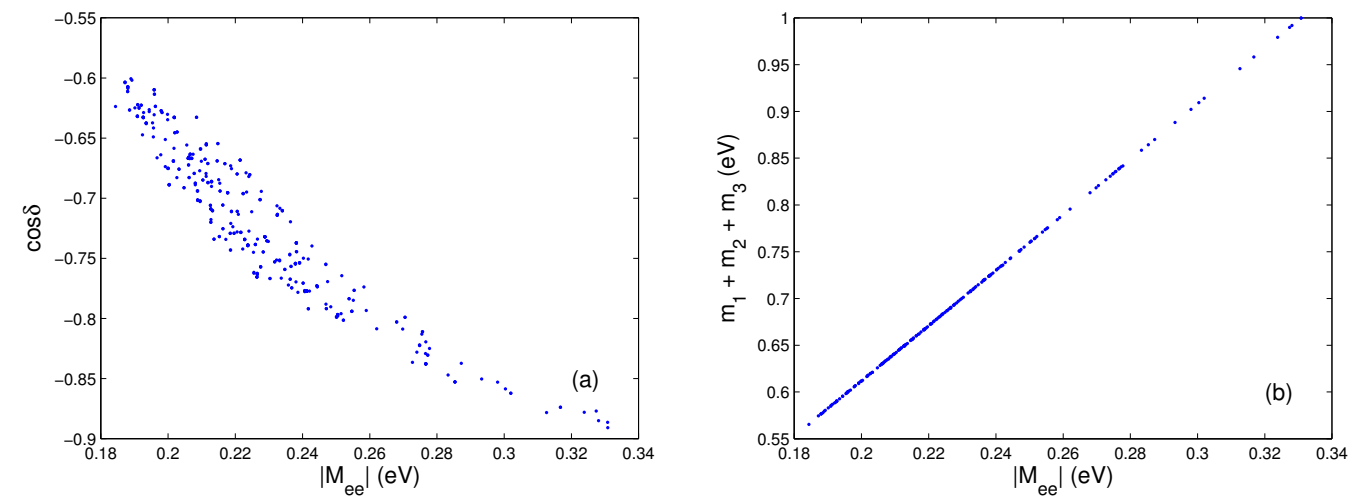

Figure 1. For model 1, we present (a) $\cos \delta$ vs. $m_{\beta \beta}$ and (b) the sum of the three neutrino masses vs. $m_{\beta \beta}$. All points shown were found requiring a $1 \sigma$ fit to the data of Fogli et al. [5]. Model 1 allows an inverted mass spectrum only.
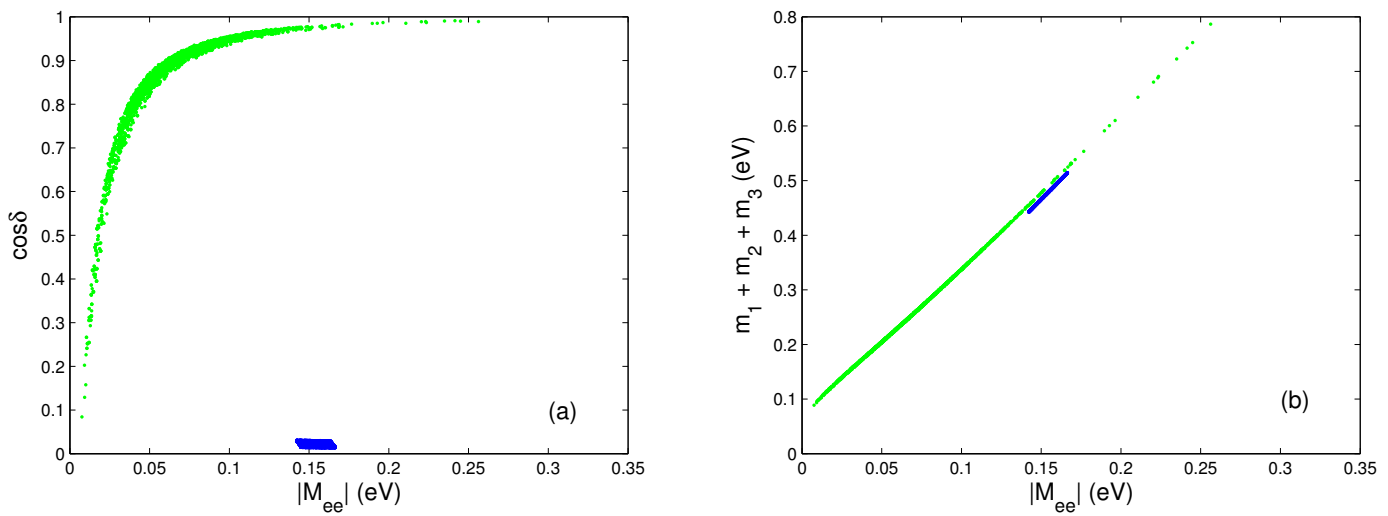

Figure 2. For model 2, we present (a) $\cos \delta$ vs. $m_{\beta \beta}$ and (b) the sum of the three neutrino masses vs. $m_{\beta \beta}$. All points shown were found requiring a $1 \sigma$ fit to the data of Fogli et al. [5]. The green (light grey) points correspond to a normal mass spectrum, while points corresponding to an inverted mass spectrum are shown in blue (black).

Model 1 is compatible only with an inverted neutrino mass spectrum. The neutrino masses must be quite high, of order $0.2 \mathrm{eV}$ or more for each neutrino, which risks violating cosmological bounds [46-48]. The phase $\delta$ is close to $\pi(\cos \delta<-0.5)$, which agrees with the (valid at the $1 \sigma$ level) preference found in ref. [5]. The corresponding scatter plots are presented as figure 1 .

For model 2 both a normal and an inverted neutrino mass spectrum are possible. However, if the spectrum is inverted then $\delta$ must be very close to $\pi / 2$ or $3 \pi / 2$ and the three neutrinos must be almost degenerate with masses around $0.15 \mathrm{eV}$, which is disfavoured by some of the recent cosmological bounds [46-48]. For a normal spectrum neutrino masses may be lower and there is a close correlation between $\cos \delta$ and the scale of neutrino masses. Notice that for model $2 \cos \delta$ is always positive, which disagrees with the findings of ref. [5] at the $1 \sigma$ level; at the $2 \sigma$ level, though, $\cos \delta$ is already unconstrained in ref. [5], so this should not be considered a severe handicap of our model. Plots for this case are given in 

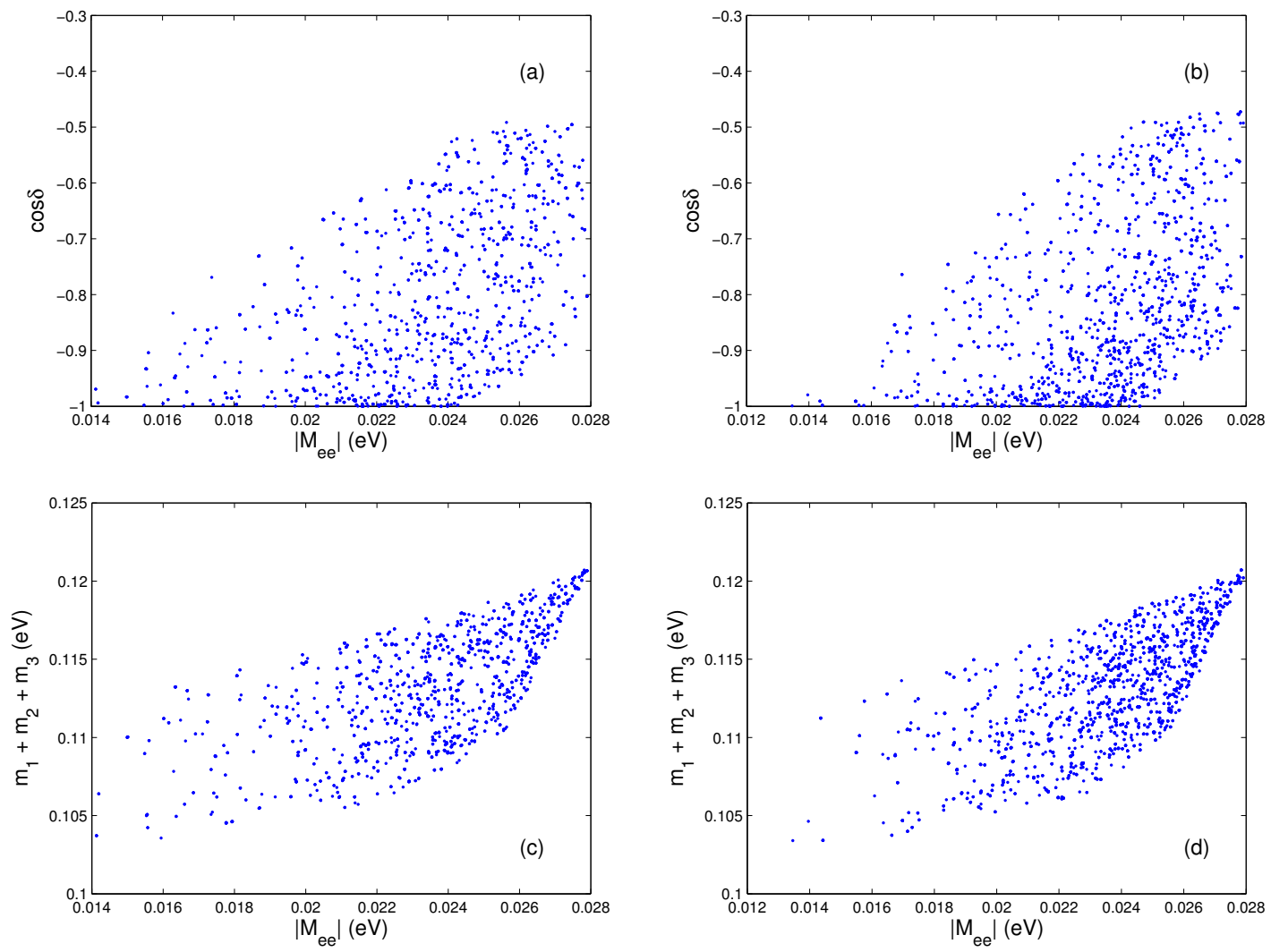

Figure 3. Plots for $\cos \delta$ vs. $m_{\beta \beta}$ in model 3 (plot a) and in model 4 (plot b); and for the sum of the neutrino masses vs. $m_{\beta \beta}$ in model 3 (plot c) and in model 4 (plot d). All points shown were found requiring a $1 \sigma$ fit to the data of Fogli et al. [5]. Models 3 and 4 allow an inverted neutrino mass spectrum only.

figure 2. Models 3 and 4 are, in practice, extremely similar. Only an inverted neutrino mass spectrum is possible for them. The neutrino masses must moreover lie in a very narrow range: the lowest mass, $m_{3}$, must lie in between $0.008 \mathrm{eV}$ and $0.012 \mathrm{eV}$. This is compatible with all current cosmological bounds. Moreover, those models force $\cos \delta<-0.4$, which is in agreement with the $1 \sigma$ range obtained in ref. [5] — see eqs. (6.3). Plots for models 3 and 4 are presented in figure 3 .

In model 5 the neutrino mass matrix $M$ is effectively real, apart from the unphysical phases of $a, b$, and $c$; neglecting those phases, $M$ may be diagonalised by a real orthogonal matrix $O$ as $O^{T} M O=\operatorname{diag}\left(m_{1},-m_{2}, m_{3}\right) \cdot{ }^{13}$ The smallest neutrino mass is $m_{3}$, since only an inverted spectrum works in model 5 . In that model the neutrino masses are exceedingly constrained: the sum of the light-neutrino masses must be $(0.110 \pm 0.003) \mathrm{eV}$ and $m_{\beta \beta}=(0.020 \pm 0.001) \mathrm{eV}$. The matrix $O$ is characterized by Dirac phase $\delta=\pi$. Besides these predictions, model 5 predicts a correlation between the mixing angles $\theta_{12}$ and $\theta_{23}$, which we depict in the scatter plots in figure 4 . One sees in figure 4 (a) that model 5 is still viable, although rather marginally, at the $1 \sigma$ level.

\footnotetext{
${ }^{13}$ We have found that only this choice of Majorana phases works: the largest eigenvalue of $M$ in absolute value, viz. $m_{2}$, must have sign opposite to the one of the other two eigenvalues.
} 

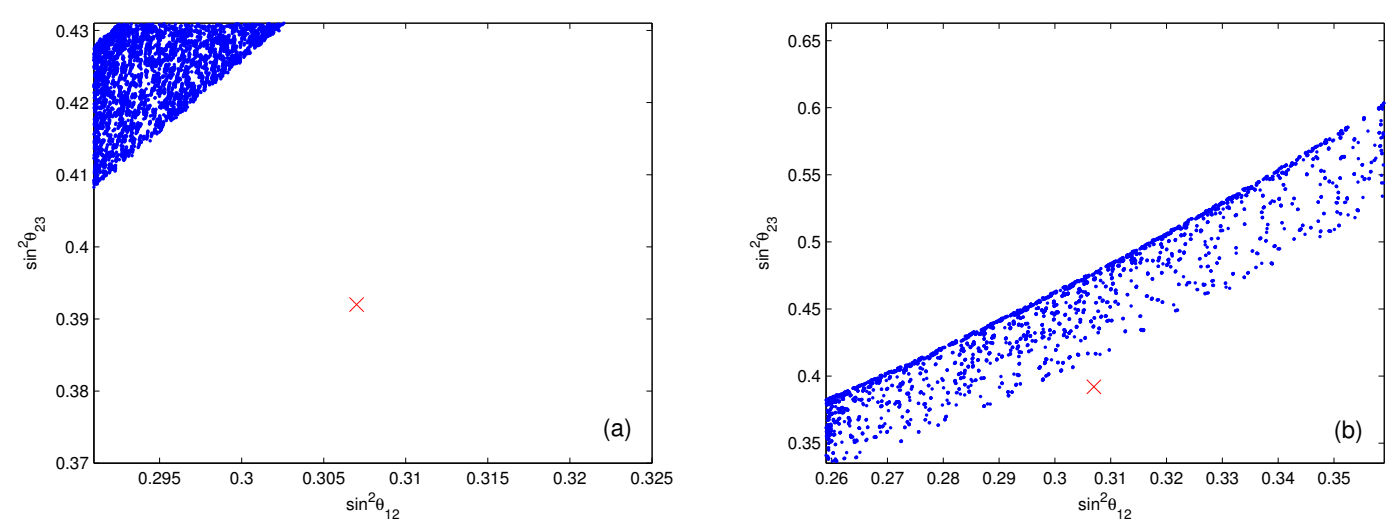

Figure 4. The correlation between $\sin ^{2} \theta_{23}$ and $\sin ^{2} \theta_{12}$ in model 5. In plot (a) all the physical quantities were forced to lie inside their $1 \sigma$ ranges of ref. [5] while in plot (b) the $3 \sigma$ ranges were used instead. The cross in both plots marks the best-fit point of ref. [5]. Model 5 only allows an inverted mass spectrum, with the Majorana phases and $\delta=\pi$ fixed (see text).

\section{Summary}

In this paper we have constructed five flavour models for the lepton sector. They are two-Higgs-doublet models with a standard $\mathbb{Z}_{2}$ symmetry which changes the sign of one of the doublets, or else with a $C P$ symmetry under which one of the doublets is odd. The models are not necessarily supersymmetric and use no soft breaking of symmetries, just spontaneous breaking. Since they are seesaw models, they include an extra high scale at which we introduce two real scalar gauge singlets and three right-handed neutrinos. Models 1-4 have five physical parameters each, while model 5 only has four parameters. They all fit very well the phenomenological data for the lepton mixing angles and for the neutrino mass-squared differences. With the exception of model 2, all our models predict an inverted neutrino mass spectrum and a negative $\cos \delta$. In models 1 and 2 the neutrino mass scale tends to be quite high, in possible conflict with cosmological bounds, but in models 3,4 , and 5 the neutrinos are fairly light. Model 5 furthermore predicts a well-defined correlation between the mixing angles $\theta_{12}$ and $\theta_{23}$.

Experimental consequences of our models may either be around the corner or unattainably far. On the one hand, the two Higgs doublets that we utilize in our models may be the ones of a two-Higgs-doublet model; either of the $\mathbb{Z}_{2}$ symmetries generated by $B$ and by $D$ in eq. (3.1) may be suitably extended to the quark sector in order to guarantee the absence of flavour-changing neutral Yukawa interactions (FCNYI) in that sector. Note that the $D_{4}$ symmetries of our models 1,3 and 5 ensure the absence of FCNYI at tree level in the lepton sector (the same statement being true for the generalized symmetries of models 2 and 4 ); the standard option [31] to avoid FCNYI in two-Higgs doublet models is a $\mathbb{Z}_{2}$ symmetry under which $\phi_{2}, e_{R}, \mu_{R}$, and $\tau_{R}$ change sign, but that option is not unique, as shown here. In our model FCNYI only arise at loop level [49]; flavour-changing couplings to the $\gamma$ and $Z^{0}$ gauge bosons are suppressed if the seesaw scale is sufficiently high [49, 50]. The FCNYI may lead to decays like $\tau^{ \pm} \rightarrow \mu^{ \pm} e^{+} e^{-}$or else to neutral-scalar decays of the 
type $S^{0} \rightarrow \tau^{+} \mu^{-}$, which may in the future be observable at the LHC; however, the flavourchanging vertices are suppressed by a loop factor and by two Yukawa couplings and will typically be small. On the other hand, the two Higgs doublets of our models may not be unique; there may be an extra Higgs doublet, call it $\phi_{0}$, which has a much larger VEV and is the only one that couples to the quarks. In such a scheme, $\phi_{1,2}$ will have large masses and small VEVs due to a type-II seesaw mechanism for Higgs doublets ${ }^{14}$ [30] and might be undetectable at the LHC.

\section{Acknowledgments}

We thank Walter Grimus for reading and commenting on a preliminary version of the manuscript. The work of PMF is supported in part by the Portuguese Foundation for Science and Technology (FCT) under contract PTDC/FIS/117951/2010, by FP7 Reintegration Grant number PERG08-GA-2010-277025, and by PEst-OE/FIS/UI0618/2011. LL is supported by Portuguese national funds through FCT project PEst-OE/FIS/UI0777/2011, and also through the projects PTDC/FIS/098188/2008, CERN/FP/123580/2011, and the Marie Curie Initial Training Network "UNILHC" PITN-GA-2009-237920. The work of POL is supported by the Austrian Science Fund (FWF) through the project P 24161-N16.

\section{References}

[1] DOUBLE-CHOOZ collaboration, Y. Abe et al., Indication for the disappearance of reactor electron antineutrinos in the Double CHOOZ experiment, Phys. Rev. Lett. 108 (2012) 131801 [arXiv:1112.6353] [INSPIRE].

[2] DAYA-BAY collaboration, F. An et al., Observation of electron-antineutrino disappearance at Daya Bay, Phys. Rev. Lett. 108 (2012) 171803 [arXiv:1203.1669] [INSPIRE].

[3] RENO collaboration, J. Ahn et al., Observation of reactor electron antineutrino disappearance in the RENO experiment, Phys. Rev. Lett. 108 (2012) 191802 [arXiv: 1204.0626] [INSPIRE].

[4] W. Grimus and L. Lavoura, Softly broken lepton numbers and maximal neutrino mixing, JHEP 07 (2001) 045 [hep-ph/0105212] [INSPIRE].

[5] G. Fogli et al., Global analysis of neutrino masses, mixings and phases: entering the era of leptonic CP-violation searches, Phys. Rev. D 86 (2012) 013012 [arXiv:1205.5254] [INSPIRE].

[6] D. Forero, M. Tortola and J. Valle, Global status of neutrino oscillation parameters after Neutrino-2012, Phys. Rev. D 86 (2012) 073012 [arXiv: 1205.4018] [INSPIRE].

[7] M. Gonzalez-Garcia, M. Maltoni, J. Salvado and T. Schwetz, Global fit to three neutrino mixing: critical look at present precision, JHEP 12 (2012) 123 [arXiv:1209.3023] [INSPIRE].

[8] W. Grimus and L. Lavoura, A nonstandard CP transformation leading to maximal atmospheric neutrino mixing, Phys. Lett. B 579 (2004) 113 [hep-ph/0305309] [INSPIRE].

\footnotetext{
${ }^{14}$ In the standard type-II seesaw mechanism, which operates on a Higgs triplet $\Delta$, there is in the scalar potential a term linear in $\Delta$, viz. $\phi^{\dagger} \Delta \tilde{\phi}$, which induces a VEV for $\Delta$ from the VEV of the Higgs doublet $\phi$. The type-II seesaw mechanism for Higgs doublets operates on a doublet $\phi^{\prime}$; a linear coupling $\phi^{\dagger} \phi^{\prime}$ in the scalar potential generates a VEV for the neutral component of $\phi^{\prime}$.
} 
[9] W. Grimus and L. Lavoura, A discrete symmetry group for maximal atmospheric neutrino mixing, Phys. Lett. B 572 (2003) 189 [hep-ph/0305046] [INSPIRE].

[10] P.H. Frampton, S.L. Glashow and D. Marfatia, Zeroes of the neutrino mass matrix, Phys. Lett. B 536 (2002) 79 [hep-ph/0201008] [INSPIRE].

[11] L. Lavoura, Zeros of the inverted neutrino mass matrix, Phys. Lett. B 609 (2005) 317 [hep-ph/0411232] [INSPIRE].

[12] W. Grimus and L. Lavoura, $\mu-\tau$ interchange symmetry and lepton mixing, Fortsch. Phys. 61 (2013) 535 [arXiv:1207.1678] [INSPIRE].

[13] W. Rodejohann, M. Tanimoto and A. Watanabe, Relating large $U_{e 3}$ to the ratio of neutrino mass-squared differences, Phys. Lett. B 710 (2012) 636 [arXiv:1201.4936] [INSPIRE].

[14] P. Ludl, S. Morisi and E. Peinado, The reactor mixing angle and CP-violation with two texture zeros in the light of T2K, Nucl. Phys. B 857 (2012) 411 [arXiv:1109.3393] [INSPIRE].

[15] G. Altarelli, F. Feruglio and L. Merlo, Tri-bimaximal neutrino mixing and discrete flavour symmetries, Fortsch. Phys. 61 (2013) 507 [arXiv:1205.5133] [INSPIRE].

[16] H. Ishimori and E. Ma, New Simple $A_{4}$ neutrino model for nonzero $\theta_{13}$ and large $\delta_{\mathrm{CP}}$, Phys. Rev. D 86 (2012) 045030 [arXiv:1205.0075] [InSPIRE].

[17] E. Ma, Self-organizing neutrino mixing matrix, Phys. Rev. D 86 (2012) 117301 [arXiv: 1209.3374] [INSPIRE].

[18] C. Lam, Finite symmetry of leptonic mass matrices, Phys. Rev. D 87 (2013) 013001 [arXiv: 1208.5527] [INSPIRE].

[19] M. Holthausen, K.S. Lim and M. Lindner, Lepton mixing patterns from a scan of finite discrete groups, Phys. Lett. B 721 (2013) 61 [arXiv:1212.2411] [INSPIRE].

[20] S.F. King, T. Neder and A.J. Stuart, Lepton mixing predictions from $\Delta\left(6 n^{2}\right)$ family symmetry, arXiv:1305.3200 [INSPIRE].

[21] C. Lam, Determining horizontal symmetry from neutrino mixing, Phys. Rev. Lett. 101 (2008) 121602 [arXiv:0804.2622] [INSPIRE].

[22] R. de Adelhart Toorop, F. Feruglio and C. Hagedorn, Finite modular groups and lepton mixing, Nucl. Phys. B 858 (2012) 437 [arXiv:1112.1340] [INSPIRE].

[23] D. Hernandez and A.Y. Smirnov, Lepton mixing and discrete symmetries, Phys. Rev. D 86 (2012) 053014 [arXiv:1204.0445] [INSPIRE].

[24] L. Lavoura, On a possible relationship between lepton mixing and the stability of dark matter, J. Phys. G 39 (2012) 025202 [arXiv:1109.6854] [InSPIRE].

[25] A. Blum, C. Hagedorn and M. Lindner, Fermion masses and mixings from dihedral flavor symmetries with preserved subgroups, Phys. Rev. D 77 (2008) 076004 [arXiv:0709.3450] [INSPIRE].

[26] P. Ko, T. Kobayashi, J.-h. Park and S. Raby, String-derived $D_{4}$ flavor symmetry and phenomenological implications, Phys. Rev. D 76 (2007) 035005 [Erratum ibid. D 76 (2007) 059901] [arXiv: 0704.2807] [INSPIRE].

[27] H. Ishimori et al., Soft supersymmetry breaking terms from $D_{4} \times Z_{2}$ lepton flavor symmetry, Phys. Rev. D 77 (2008) 115005 [arXiv:0803.0796] [INSPIRE]. 
[28] C. Hagedorn and R. Ziegler, $\mu-\tau$ symmetry and charged lepton mass hierarchy in a supersymmetric $D_{4}$ model, Phys. Rev. D 82 (2010) 053011 [arXiv:1007.1888] [INSPIRE].

[29] D. Meloni, S. Morisi and E. Peinado, Stability of dark matter from the $D_{4} \times Z_{2}$ flavor group, Phys. Lett. B 703 (2011) 281 [arXiv: 1104.0178] [inSPIRE].

[30] W. Grimus, L. Lavoura and B. Radovcic, Type II seesaw mechanism for Higgs doublets and the scale of new physics, Phys. Lett. B 674 (2009) 117 [arXiv:0902.2325] [INSPIRE].

[31] G. Branco et al., Theory and phenomenology of two-Higgs-doublet models, Phys. Rept. 516 (2012) 1 [arXiv:1106.0034] [INSPIRE].

[32] P.M. Ferreira, R. Santos, M. Sher and J.P. Silva, Implications of the LHC two-photon signal for two-Higgs-doublet models, Phys. Rev. D 85 (2012) 077703 [arXiv:1112.3277] [INSPIRE].

[33] S. Chang et al., Comprehensive study of two Higgs doublet model in light of the new boson with mass around $125 \mathrm{GeV}$, JHEP 05 (2013) 075 [arXiv:1210.3439] [INSPIRE].

[34] G. Bélanger, B. Dumont, U. Ellwanger, J. Gunion and S. Kraml, Higgs couplings at the end of 2012, JHEP 02 (2013) 053 [arXiv:1212.5244] [INSPIRE].

[35] C.-Y. Chen and S. Dawson, Exploring two Higgs doublet models through Higgs production, Phys. Rev. D 87 (2013) 055016 [arXiv: 1301.0309] [INSPIRE].

[36] ATLAS collaboration, Observation of a new particle in the search for the standard model Higgs boson with the ATLAS detector at the LHC, Phys. Lett. B 716 (2012) 1 [arXiv: 1207.7214] [INSPIRE].

[37] CMS collaboration, Observation of a new boson at a mass of $125 \mathrm{GeV}$ with the CMS experiment at the LHC, Phys. Lett. B 716 (2012) 30 [arXiv:1207.7235] [INSPIRE].

[38] W. Grimus and L. Lavoura, The Seesaw mechanism at arbitrary order: disentangling the small scale from the large scale, JHEP 11 (2000) 042 [hep-ph/0008179] [INSPIRE].

[39] M. Holthausen, M. Lindner and M.A. Schmidt, CP and discrete flavour symmetries, JHEP 04 (2013) 122 [arXiv: 1211.6953] [INSPIRE].

[40] F. Feruglio, C. Hagedorn and R. Ziegler, Lepton mixing parameters from discrete and $C P$ symmetries, JHEP 07 (2013) 027 [arXiv: 1211.5560] [INSPIRE].

[41] G. Ecker, W. Grimus and W. Konetschny, Quark mass matrices in left-right symmetric gauge theories, Nucl. Phys. B 191 (1981) 465 [INSPIRE].

[42] G. Ecker, W. Grimus and H. Neufeld, Spontaneous CP-violation in left-right symmetric gauge theories, Nucl. Phys. B 247 (1984) 70 [INSPIRE].

[43] P. Ferreira, W. Grimus, L. Lavoura and P. Ludl, Maximal CP-violation in lepton mixing from a Model with $\Delta(27)$ flavour symmetry, JHEP 09 (2012) 128 [arXiv:1206.7072] [INSPIRE].

[44] R. Mohapatra and C. Nishi, $S_{4}$ flavored CP symmetry for neutrinos, Phys. Rev. D 86 (2012) 073007 [arXiv: 1208. 2875] [InSPIRE].

[45] Particle Data Group collaboration, J. Beringer et al., Review of particle physics, Phys. Rev. D 86 (2012) 010001 [inSPIRE].

[46] R. de Putter et al., New neutrino mass bounds from Sloan Digital Sky Survey III data release 8 photometric luminous galaxies, Astrophys. J. 761 (2012) 12 [arXiv:1201.1909] [INSPIRE].

[47] Z. Hou et al., Constraints on cosmology from the cosmic microwave background power spectrum of the 2500-square degree SPT-SZ Survey, arXiv:1212.6267 [INSPIRE]. 
[48] Planck collaboration, P. Ade et al., Planck 2013 results. XVI. Cosmological parameters, arXiv: 1303.5076 [INSPIRE].

[49] W. Grimus and L. Lavoura, Soft lepton flavor violation in a multi Higgs doublet seesaw model, Phys. Rev. D 66 (2002) 014016 [hep-ph/0204070] [INSPIRE].

[50] R. Alonso, M. Dhen, M. Gavela and T. Hambye, Muon conversion to electron in nuclei in type-I seesaw models, JHEP 01 (2013) 118 [arXiv:1209.2679] [INSPIRE]. 